\title{
EL INCA GARCILASO DE LA VEGA, SÍMBOLO AUTÉNTICO DE PERUANIDAD
}

\begin{abstract}
RESUMEN
El Inca Garcilaso de la Vega es el prosista más destacado de las letras hispanoamericanas de la Colonia, el primer escritor mestizo y precursor del Indigenismo en la literatura peruana. Con su pluma magistral y dotes de historiador recreó diferentes aspectos del antiguo Perú, siempre con un espíritu integrador, capaz de fusionar a indios, mestizos y criollos. Su madre y sus parientes maternos se encargaron de enseñarle el quechua. Le hicieron conocer mediante historias maravillosas toda la grandeza del Imperio de los Incas. Así por sus ojos de niño inquieto, pasaron los sucesos guerreros, las costumbres y tradiciones imperiales del Tahuantinsuyo, que evocará con nostalgia en su creación literaria. Su obra los Comentarios reales fue escrita con plena identificación peruanista, contiene los ricos archivos de la historia peruana, siendo el monumento más noble que Garcilaso pudo erigir a la memoria de sus antepasados, uniendo su vocación de historiador y alma de poeta. Así, los Comentarios reales constituye la expresión más perfecta del mestizaje étnico y cultural porque representa la síntesis de dos mundos diferentes: incaico y español, dando una imagen del Imperio Incaico y primeros años de Conquista con estilo
\end{abstract}

Carmen Irma Samanez Paz ${ }^{1}$

elegante y vigoroso, lleno de sensibilidad e imaginación. Los Comentarios reales es la expresión sincera del afecto que sintió por sus antepasados los incas y motivo de tributo a su raza indígena materna. En esta obra perfila la exacta dimensión del nuevo Perú y asoma la naciente conciencia nacional, razón por la cual los estudiosos consideran a Garcilaso el primer escritor de nuestra historia que pretende reivindicar las glorias del Tahuantinsuyo, llegando a ser un auténtico símbolo de la peruanidad, capaz de entregarnos páginas llenas de evocación y melancolía por el antiguo Perú. El conocimiento y compresión de su obra nos acerca a Garcilaso, peruano de corazón y cronista mestizo que nos llena de orgullo. Su mensaje resulta trascendental para todos por su peruanismo y dimensión universal. Los Comentarios reales es una obra trabajada con tenacidad y constancia que reclama una lectura cuidadosa por la variedad de sus enfoques, la brillantez y originalidad de su estilo y la hondura interpretativa del Perú en tiempos remotos. Fue el más grande escritor del período Colonial, no solo fue un cronista, un comentarista de crónicas ajenas, sino un historiador interesado por todos los aspectos de la vida humana, capaz de examinar la

1 Licenciada en Educación en la especialidad de Lengua y Literatura, Directora del Departamento Académico de Lingüística, Profesora Principal de la Universidad Nacional de San Antonio Abad del Cusco. E-mail: carmensamanezpaz@hotmail.com 
realidad desde diferentes ángulos, hecho que hace de su producción literaria una verdadera joya artística e histórica.

PALABRAS CLAVES: Mestizo, peruanidad, estirpe, cronista, nacionalidad, legado étnico-cultural, poético, simbiosis.

\section{ABSTRACT}

The Inca Garcilaso de la Vega is the most prominent prose writer of the Spanish-American letters of the Colony, the first mestizo writer and precursor of Indigenismo in Peruvian literature. With his masterful pen and historian's talents he recreated different aspects of ancient Peru, always with an integrating spirit, capable of fusing Indians, mestizos and creoles. His mother and his maternal relatives were in charge of teaching him Quechua. They made him known through wonderful stories all the greatness of the Inca Empire. Thus by the eyes of a restless child, passed the warlike events, customs and imperial traditions of the Tahuantinsuyo, which will evoke with nostalgia in his literary creation. His work The Real Commentaries was written with full Peruvian identification, contains the rich archives of Peruvian history, being the most noble monument that Garcilaso could erect to the memory of his ancestors, uniting his vocation as a historian and poet's soul. Thus, the Real Commentaries constitutes the most perfect expression of the ethnic and cultural mestizaje because it represents the synthesis of two different worlds: Inca and Spanish, giving an image of the Inca Empire and first years of conquest, with elegant and vigorous style, full of sensitivity and imagination. The Real Comments is the sincere expression of the affection felt by the ancestors of the Incas and a source of tribute to their native Indian race. In this work he outlines the exact dimension of the new Peru and the nascent national consciousness appears, which is why scholars consider Garcilaso the first writer of our history who claims to claim the glories of Tahuantinsuyo, becoming a true symbol of Peruvian, Capable of delivering pages full of evocation and melancholy for ancient Peru. The knowledge and understanding of his work brings us closer to Garcilaso, a Peruvian of heart and a mestizo chronicler who fills us with pride. His message is transcendental for all by its Peruvian and universal dimension. The Real Commentaries is a work worked with tenacity and constancy that demands a careful reading for the variety of its approaches, the brilliance and originality of its style and the interpretive depth of Peru in remote times. He was the greatest writer of the colonial period, not only a chronicler, a commentator of other chronicles, but a historian interested in all aspects of human life, capable of examining reality from different angles, a fact that makes his literary production A true artistic and historical jewel.

KEYWORDS: Mestizo, Peruvian, race, chronicler, nationality, ethnic-cultural legacy, poetic, symbiosis.

$\mathrm{L}$ a ciudad del Cusco en cuanto a su $\checkmark$ cultura literaria, es cuna de grandes escritores, ya sea narradores y poetas que supieron calar en el alma del pueblo cusqueño y han aportado un valioso legado intelectual y artístico, convirtiéndose en un baluarte cultural. Así el Cusco en su vasto y fecundo proceso histórico, social, político y cultural, ha dado lugar a grandes personalidades literarias como Narciso Aréstegui, Clorinda Matto de Turner, Luis Nieto Miranda, Inca Garcilaso de la Vega, entre otros.

Al conmemorar el IV Centenario del Fallecimiento del Inca Garcilaso de la Vega, queremos rendirle un tributo de amor y admiración a este destacado precursor del Indigenismo en la literatura peruana, primer mestizo peruano, símbolo de peruanidad, el más sobresaliente cronista postoledano que representa la síntesis de dos mundos diferentes: el incario y el español. Es el narrador más sorprendente y más influyente en las actuales generaciones que siguen sus pasos en el campo literario.

La vida de este insigne escritor está íntimamente vinculada a su producción literaria; por cuanto parte del contenido de su creación es producto de sus vivencias, experiencias, inquietudes, preocupaciones y de su concepción vital que supo reflejar con gran talento. Todo lo que vivió, oyó y leyó, le ha impulsado a escribir, a manera de imperiosa necesidad; debido a que su producción literaria tiene un carácter autobiográfico, porque se nutrió de todas las acciones vitales que cosechó a lo largo de su intrincada vida.

Los Comentarios reales es considerada por la crítica como la obra maestra del Inca Garcilaso de la Vega, libro de cultura que presenta una visión integral de nuestros antepasados los incas, fue la fuente más representativa de la historiografía peruana, con una gran influencia universal en escritores de diversas literaturas interesados por la América precolombina y la dominación colonial. Es la obra que seduce a propios y extraños que encierra la conciencia nacional, siendo una verdadera expresión espiritual del Perú. A lo largo de sus animadas páginas del gran cusqueño, se advierte al mismo tiempo el espíritu indigenista, mestizo, criollo y peruanista. Sin duda alguna es el alma máter de la auténtica peruanidad y del más acendrado 
cusqueñismo. Al leer los Comentarios reales nos sentimos orgullosos, identificándonos con esa emoción del pasado lleno de majestad y grandeza, nos sirve para tomar conocimiento de nuestro Perú, nos invita a buscar nuestra cultura y pasado, porque se trata de la obra más fecunda que nos permite nutrirnos del espíritu nacional, puesto que sus páginas están llenas de recordación y amor por nuestra tierra, con una revaloración de usos, tradiciones, costumbres, formas de vida e idiosincrasia propia del cusqueño. Además el autor fue reconocido por sus conocimientos diversos sobre la flora y la fauna de nuestra región.

Esta monumental producción literaria fue escrita en base a los recuerdos lejanos de la niñez y juventud de Garcilaso; por cuanto nuestro escritor pudo gozar de las fiestas tradicionales incas que se seguían conservando, porque muchos indios ancianos continuaban apegados a sus costumbres. Todas las historias y leyendas fabulosas contadas por sus parientes maternos, entre ellos su tío Francisco Huallpa Túpac, su tío abuelo Inca Cusi Huallpa y los viejos capitanes Juan Pechuta y Chauca Rimachi, ambos generales heroicos del incanato, quienes le informaron a Garcilaso sobre la grandeza del Imperio Incaico. Así en el alma del inca, latía el recuerdo de su patria añorada y con la ayuda de las evocaciones guardadas en su prodigiosa memoria, decidió escribir un libro forzado de la identificación con su tierra, completando con los datos enviados por visitantes que habían venido al Cusco, así como la recopilación de informaciones remitidas por sus amigos y parientes del Perú, cartas de Diego de Alcobaza (hijo de su ayo Juan), de García Sánchez de Figueroa, de Fray Luis Jerónimo de Oré y otros colaboradores anónimos; pues sabemos que los Comentarios reales mayormente escribió en Córdoba, en la parroquia Santa María La Mayor, donde fijó su residencia cuando dejó Montilla, producción literaria que fue prohibida por el Consejo de Indias por considerarla fuente peligrosa para los indígenas del Virreinato del Perú.

Luego de haber realizado el acopio de datos, el Inca Garcilaso de la Vega completó su información con los datos recopilados de sus lecturas y testimonios de los cronistas Pedro Cieza de León, José Acosta, López de Gómara, Juan Polo de Ondegardo, Agustín de Zárate, mereciendo especial atención el cronista y jesuita peruano Blas Valera, cuya obra fue leída con devoción por Garcilaso. De esta manera nuestro escritor inicia su intenso y variado conocimiento sobre el origen de los incas, las formas de gobierno, la organización social, las leyes, la religión, los cultos, la música, la literatura, el trabajo agrícola, la medicina, la educación, la arquitectura, el sistema hidráulico y las fiestas y costumbres propias del Imperio Incaico; dando lugar a la Primera Parte de los Comentarios reales, que publicó en Lisboa en 1609, parte integrada por nueve libros.

Posteriormente y con ese entusiasmo que nunca decayó, elaboró la Segunda Parte, bajo la denominación de Historia general del Perú que publicó en Córdoba en 1617, parte compuesta por ocho libros, en los que Garcilaso narra la llegada de los españoles, las peripecias de la conquista, las amargas Guerras Civiles entre pizarristas y almagristas, la repartición de tierras, el castigo y levantamiento de los tiranos, así como el gobierno de los primeros virreyes hasta llegar al de Francisco Toledo. De esta manera, nuestro autor destaca el profundo amor y los grandes valores que tuvo por su cuna materna completada con la penetración de la cultura de occidente. No debemos olvidar que Cusco fue la capital del Tahuantinsuyo y cuna de la maravillosa y extraordinaria civilización incaica, donde la raza autóctona sufrió intensamente el impacto de la conquista española, con su secuela de servidumbre y vasallaje. Esos momentos sangrientos y dolorosos son evocados con nostalgia por Garcilaso, quien luego de experimentar un hondo 
sentimiento de emoción y orgullo por pertenecer a la gloriosa y guerrera raza india, sintió angustia y frustración, motivo por el cual se expresó: "Trocóse nuestro reinar en vasallaje", dándonos a entender el cambio de suerte de sus antepasados, quienes pasaron de la grandeza al sometimiento.

De este modo, surge la recia figura del Inca Garcilaso de la Vega, escritor consagrado cuya fama alcanzó repercusión internacional porque su voz ha trascendido las fronteras regionales y nacionales para alcanzar una dimensión universal y por tanto, su obra narrativa nos llena de satisfacción, permaneciendo como un hito de aporte valioso a la literatura peruana, siendo el primer cronista mestizo peruano que se preocupó por sentar la base indígena del antiguo Perú, además de ser considerado el iniciador de la peruanidad en nuestras letras. La fuerte preocupación que sintió Garcilaso por los problemas socio-políticos de su tiempo jugaron un papel importante en su narrativa y se convirtieron en el centro de interés o leit motiv de su cosmovisión andina, pues su creación encierra una afirmación de lo nuestro, de lo nativo y de lo mestizo; es decir, tiene un sesgo indigenista que lo hace muy peculiar. Amaba profundamente el paisaje serrano y modos de vida del Cusco que asimiló con avidez.

La grandeza del Inca Garcilaso de la Vega no solo radica en lo que pudo revelar sobre las ideas de su tiempo, sino también en su magistral uso del lenguaje renacentista, con clara dicción y presencia de muchos vocablos quechuas, idioma que aprendió durante los años de su infancia al criarse cerca a su madre. Diremos que su estilo es original, sobrio y a veces vigoroso, habiendo elaborado una auténtica joya narrativa de carácter artístico e histórico que desafía el tiempo. En los diversos episodios que relata se muestra como un autor bien documentado y audaz para darnos una visión holística del Imperio Incaico antes y después de la Conquista, abarcando hasta las Guerras Civiles, que duraron muchos años.

Su prosa se caracteriza por ser armoniosa y serena con matices elegantes acompañados de una fina ironía. Los hechos que relata son minuciosos y ordenados con evidente predominio del tempo lento, buscó la exactitud y claridad en su expresión, demostrando a su vez gran lucidez para el análisis de los sucesos descritos; denota gusto por la simetría y por lo clásico, revelando su formación de carácter humanístico, obtenido gracias a su contacto con círculos jesuitas. Estando en Montilla y Extremadura, Garcilaso asimiló una vasta cultura europea, ya que se encontraba en pleno apogeo renacentista, hecho que refleja en su producción literaria que escribió con cariño peruanista. Al respecto, José de la Riva Agüero dice que:

"Tenía el don de narrar para animar los datos más fríos de la historia”.

Algunos críticos le han atribuido a Garcilaso una tendencia imaginativa de fuerte influencia neoplatónica cuando pondera las hazañas de los incas, señalando que idealizó la gloria del Tahuantinsuyo y exaltó la grandeza de su raza, reivindicando su orgullo de ser indio, porque solo destacó los méritos y las bondades de los hijos del Sol, obviando los defectos. Lo cierto es que la prosa de Garcilaso es magistral, con lujo de detalles que destaca enfáticamente, debido a que lleva una cuenta pormenorizada de los hechos que relata con gran amenidad, exquisita sensibilidad, vuelo imaginativo y fuerte carga afectiva al evocar el pasado. Por ello, Luis Alberto Sánchez dice que: "Los Comentarios reales es un maravilloso castillo de melancolías" y Antonio Cornejo Polar manifiesta que la obra príncipe de Garcilaso: "Es una crónica idealizada que armoniza el Incario y la Conquista"; mientras que Alberto Tauro sostiene que Garcilaso es: "El hombre de la Colonia que recoge todo legado indígena y lo funde con el espíritu español de la Conquista". 
Su lenguaje posee fuerza expresiva y poder evocador, haciendo hincapié a las inquietudes sociales, culturales y políticas propias del siglo XVI. Nuestro autor con pleno conocimiento relata el levantamiento de Hernández Girón, viendo con pena cuando su padre fue entregado como rehén. Además fue testigo de las ejecuciones y duelos de honor, tan comunes en esos tiempos. Conoció personalmente a Gonzalo Pizarro, a Diego Centeno y a Francisco de Carbajal, "El Demonio de los Andes". Su obra medular contiene los más ricos archivos de la historia peruana, llegando a ser el cronista genial y la autoridad histórica decisiva en las letras hispanoamericanas de la Colonia porque recreó cuadros del antiguo Perú, enfocando con tono apasionado la vida del Incario. Su visión es profunda y cautivante, fusionó sus dos vidas de indio y español con espíritu integrador. Por esta razón, Raúl Porras Barrenechea le denomina al autor: "Español en el Perú, indio en España" porque vivió a plenitud el Incanato, uniendo a su vocación de historiador su alma de poeta.

Podemos afirmar que su obra los Cometarios reales es fruto del gran amor que sintió Garcilaso por sus antepasados incas como españoles, en la que refleja la exacta dimensión del nuevo Perú y asoma la naciente conciencia nacional. Se le considera al autor como el primer crítico de nuestra historia que defendió con ardor a su raza y a su pueblo, el cual con sabiduría forjó una gran cultura que fue destruida por los conquistadores. El contexto histórico del autor se caracterizó por ser una etapa de luchas intestinas y enfrentamientos porque la ambición y codicia de los españoles fue apoderarse del oro. Referente a ello, el mismo Garcilaso recuerda que el Cusco vivió: "Entre armas y caballeros, entre sangre y fuego de las guerras que entonces hubo".

Producida la conquista del Perú, se realizó el proceso de transculturización, el castellano en contacto con el quechua, aymara y otros dialectos, sufrió natural influencia. Por su parte, los españoles aprendieron el quechua como herramienta de conquista, que les sirvió para catequizar y destruir las idolatrías de los incas. Así nuestro cronista tomó la pluma para dar su particular visión de la historia de los incas y la conquista; debido a que el Tahuantinsuyo sucumbió ante las armas españolas. Del enfrentamiento entre estas dos civilizaciones, nació una nueva cultura, creada gracias a los aportes de ambos mundos que gravitaron en la lucha interna que marcó fuertemente las experiencias de Garcilaso durante su existencia.

El Perú del siglo XVI transcurrió entre el avance del ejército español, la resistencia de los indígenas y posteriormente, las guerras entre los mismos conquistadores; surgiendo como forma de expresión las crónicas que fueron producto del mestizaje de aquellos años de la Conquista. Su obra emblemática los Comentarios reales fue escrita como sostiene el mismo Garcilaso para enmendar los errores de los anteriores cronistas. Según la valoración que hace Ricardo González Vigil: "Es una de las cumbres indudables del genio literario de Garcilaso".

De esta manera, nuestro Inca Garcilaso de la Vega surge con fuerza en el campo literario como un genio intelectual de las letras peruanas, siempre tuvo el deseo de volver al Perú, su amada tierra, hecho que no fue posible por falta de medios económicos. La vida de nuestro ilustre cusqueño y figura sobresaliente del pensamiento ilustrado del siglo XVI ya tocaba a su fin, luego de haber llevado una vida intensa, vivió como escribió él mismo en: "Los rincones de la soledad y la pobreza donde pasó una vida quieta y pacifica, un hombre desengañado y despedido de este mundo y de sus mudanzas", dedicándose a la lectura de libros que enriquecieron su mente y dado a la oración, porque luego se ordenó de clérigo. 
Así, la presencia histórica del Inca Garcilaso de la Vega ha merecido especial atención de estudiosos e investigadores del mundo que han aquilatado el valor de su creación literaria nacida de las más auténticas entrañas cusqueñas, siendo alabado por las instituciones culturales, científicas y sociales, por la vigencia y vitalidad de sus pensamientos sobre el Perú antiguo. Su repercusión literaria hizo posible que sea considerado en casi todos los libros de literatura, pues su solo nombre llevó a la cúspide el campo narrativo. Hoy en día los Comentarios reales y su autor gozan del prestigio universal y es fuente de consulta obligatoria. Con justificada razón Jorge Basadre señala que su obra: "Es el cantar de gesta de la nacionalidad".

Su obra representativa los Comentarios reales ha sido apreciada con mayor fuerza durante las últimas décadas y pese a su muerte acaecida el 23 de abril de 1616, su luz no se extingue y está presente en nosotros porque el Inca Garcilaso de la Vega fue y será una figura extraordinaria. Todos los cusqueños tenemos la obligación de saber quién fue y qué hizo este personaje de las letras peruanas, el mismo que nos dejó un mensaje de peruanidad con proyección futura, pues su inquietud de hombre inconforme hizo que anhelara propósitos nobles para su amada patria.

Un episodio que merece destacar en Garcilaso por su calidad artística es su plena identificación con el Cusco, su tierra natal, demostrando intenso afecto por todo lo que le rodeaba a esta Ciudad Imperial, describió con vehemencia y tinte melancólico su antigua casa, patios empedrados, caballerizas, andenes sorprendentes, tambos estratégicos, piedras milenarias, punas desoladas, apus sagrados, callecitas típicas y zonas arqueológicas que se convirtieron en motivos del ambiente paisajístico en los relatos maravillosos y reflexivos provenientes de sus experiencias totales que ponen al descubierto su amor por la patria que lo vio nacer, cuyo aliento y verdad dejan entrever el testimonio de un hombre sufrido, sensible e imaginativo y desde esta perspectiva brotó su manifestación en forma natural al señalar: "Al Cuzco lo llevo en mi alma y lo siento en mi corazón", afirmando de esta manera la gratitud hacia su pueblo milenario con la esperanza de fortalecer su identificación plena con su raza india.

Por todo lo expuesto, el Inca Garcilaso de la Vega tiene ya un lugar privilegiado en la narrativa castellana de todos los tiempos y el primero en la literatura peruana porque puso su vida al servicio de un ideal: el de lograr un nuevo Perú.

\section{REFERENCIAS}

AVALLE-ARCE, Juan Bautista (1970) El Inca Garcilaso en sus "Comentarios". Madrid, Editorial Gredos.

BULNES, José (1967) Estructura de los Comentarios reales. Lima, Universidad Mayor de San Marcos.

FLORES GALINDO, Alberto (1988) Buscando un inca: Identidad y utopía en los Andes. Lima, Editorial Horizonte.

GONZALEZ VIGIL, Ricardo (1989) Genio y complejidad del Inca Garcilaso. Lima, Dominical suplemento de El Comercio.

LAVALLE, Bernard (1982) El Inca Garcilaso de la Vega. Madrid, Editorial Cátedra.

PORRAS BARRENECHEA, Raúl (1946) El Inca Garcilaso de la Vega. Lima, Editorial Lumen.

TAURO, Alberto (1985) Biografía del Inca Garcilaso. Lima, Banco de Crédito del Perú.

VARCÁRCEL, Luis (1939) Garcilaso el Inca, visto desde el ángulo indio. Lima, Imprenta del Museo Nacional.

YÉPEZ MIRANDA, Alfredo (1955) Garcilaso: símbolo del americano. Lima, Banco de Crédito. 